\title{
Channel-Aware Scheduling Algorithms with Channel Prediction for Reliable Multicast Data Transmission over Geostationary Satellite Networks
}

\author{
*A.Sali, *G.Acar, *B.Evans \\ *Center for Communication Systems Research (CCSR), \\ University of Surrey, \\ GU2 7XH Guildford, UK \\ \{A.Sali, G.Acar, B.Evans\}@surrey.ac.uk
}

\author{
G. Giambene \\ CNIT-Università degli Studi di Siena, \\ Via Roma, 56, \\ 53100 Siena, Italy \\ giambene@unisi.it
}

\begin{abstract}
In this paper, Real-Time Channel Prediction (RTChP) technique is implemented with channel-aware scheduling algorithms to increase decision accuracy at the scheduler. The channel-aware scheduler receives feedback messages in the form of Channel State Information (CSI) from the terminals via reservation slots in the return link. In the face of reliable multicast transmission, the channel-aware scheduler aims to reduce the number of packets received in error at the terminals. As a consequence, additional retransmission passes can be decreased, resulting in higher resource utilization in the forward link and reduced session delay. Simulation results are presented with considerations of a Channel-Aware Scheduling (CAS) algorithm. From simulation results, RTChP increases performance gain when a lower number of terminals are considered. However, when the scenario involves more terminals, the prediction technique is ineffective and its performance deteriorates. Essentially, the RTChP implemented at the scheduler is useful only for small multicast group.
\end{abstract}

Keywords - real-time channel prediction technique, channelaware scheduling algorithms, reliable multicast transmission, geostationary satellite networks

\section{INTRODUCTION}

Multicast services are well supported by geostationary satellites as a result of their inherent broadcasting capabilities over large coverage areas. However, in the context of reliable transmission, its configuration and management must be accurately analysed, since satellite resources are expensive and link quality degrades significantly during fading. Also, in transmission of multicast data from a satellite direct to $N$ receivers, repeated retransmissions due to bad channel conditions may exhaust the forward link capacity, as reported in [1]. This problem is more pronounced in case of large multicast groups since the performance of the communication depends on the performance of a group of users that are possibly located across the spotbeam, hence experiencing different channel conditions.

There are possibilities of tackling user heterogeneity in multicast transmission. One of them, which are getting more attention, is cross-layer design. Cross-layer design suggests (possibly joint) adaptation of communication protocols and mechanisms at various layers according to the information collected at other layers of the communication system. Recent growth in heterogeneous networks entails adaptive mechanisms. In this framework, a cross layer approach would be more effective and flexible. In wireless systems where both radio resources and power are strictly constrained, resource optimisation is needed when such opportunity is not guaranteed by the strictly layered protocol stack.

In this paper, we implement cross-layer approach at a channel-aware scheduler in Radio Access Network (RAN). Specifically, Channel State Information (CSI) from physical layer (PHY) of the terminals is reported via return link for the scheduler's utilization which resides in Medium Access Control (MAC) of (RAN). To increase the accuracy of scheduling decisions, the integration of a channel prediction technique with the channel-aware scheduling (CAS) algorithm is proposed and investigated in this paper. In return, resource utilisation will be increased and session delay reduced. The challenge with respect to the scheduling design arises from the fact that a multicast scenario is considered, where scheduling decision concerns all users in the multicast group. In other words, the fluctuating channel condition from a large number of users has to be considered, whilst optimizing scheduling decision to benefit all users as well as network operator The scheduling algorithm allows an efficient transmission of multicast data by means of reacting to users' channel condition via updating scheduling parameters to control the transmission of packets in the forward link.

The reference system is based on an $L$-Band geostationary satellite network representing Inmarsat BGAN (Broadband Global Area Network) system shown in Figure 1. The scheduling is executed at the Radio Access Network (RAN)

* Corresponding author. Address: Centre for Communication Systems Research, University of Surrey, Guildford, GU2 7XH, United Kingdom, tel. no. ++44 1483683036 , fax. no. ++44 1483686001

Email addresses: A.Sali@surrey.ac.uk (A. Sali), G.Acaar@surrey.ac.uk (G. Acar), B.Evan@surey.ac.uk (B. Evans), giambine@unisi.it (G. Giambene). This paper has been carried out within the framework of the European SatNEx II (contract No. IST-027393, network of excellence, www.satnex.org, joint activity JA2330. 
and a geostationary satellite relays multicast data products from Multicast File Transport Protocol (MFTP) server to all multicast receivers via $N$ number of Mobile Terminals (MT) and MFTP Client termed as users or receivers. The reliable transmission under consideration outlines that all terminals receive the exact copy of the transmitted file. The multicast reliability is achieved via a multicast transport protocol with a retransmission strategy that is based on StarBurst MFTP (Multicast File Transfer Protocol) [3]. The relation between file, MFTP block and Data Transmission Unit (DTU) is shown in Figure 2. According to MFTP, a multicast file is first transmitted to all group members in its entirety in the initial pass. In response, after the end of the original transmission pass, receivers send negative acknowledgments (NACK) indicating lost DTUs. If any DTU retransmissions are required, then the MFTP server makes another pass (i.e. pass number 2) through the file, but sends only those DTUs that were reported as missed by at least one client. Additional passes may be required to successfully deliver all DTUs to all MFTP clients. In essence, the retransmission passes increases with the number of terminals and slot error rate.

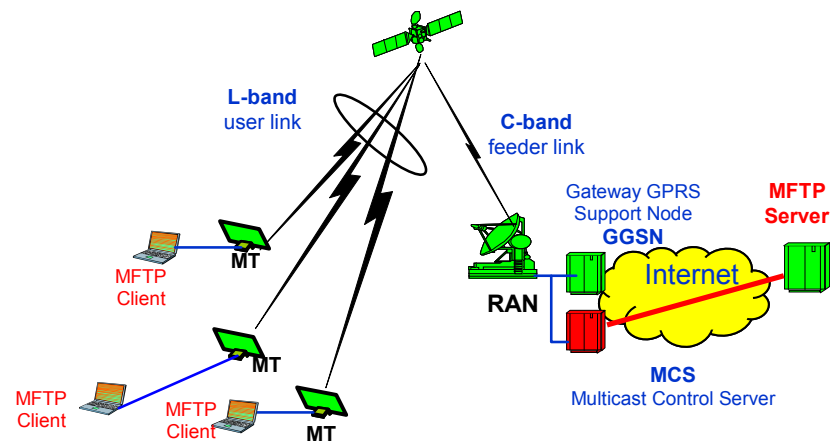

Figure 1. Reference system architecture. The satellite provides broadband access for transmission of multicast data product to receiver

If a terminal received a Data Transmission Unit (DTU) unsuccessfully, then the DTU will be retransmitted. This scenario not only reduces resource utilization in the forward link, but also increases session delay because of the retransmission process. Note that for each retransmission pass, one round trip time is added to the session delay. Hence, the aim of utilizing CSI updates from terminals by the scheduler in RAN is to increase forward link resource utilization and reduce session delay. To increase the accuracy of scheduler decision, it is expected that the performance of the scheduler is further improved with real-time channel prediction technique (RTChP).

The propagation environment is in the range of $L$-band channel model based on parameters from [2]. The propagation parameters are recommended values to match the extracted time-series parameters from measurements. Basically, the channel model makes use of a two-state semi-Markov model representing Line-Of-Sight (LOS) and fading states. The propagation parameters boil down to bit energy per noise density, $E_{b} / N_{o}$. From the value of $E_{b} / N_{o}$, the appropriate bit error rate, $\phi$, can be found according to the specified coding and modulation parameters. The terminal then calculates its respective forward bearer slot error rate, $p$, as a function of BER and payload size, $L$ :

$p=1-(1-\phi)^{L}$

The forward bearer slot error rate, $p$, is the Channel State Information (CSI) parameter of interest in our work. Herewith the forward bearer slot error rate will simply be referred as slot error rate.

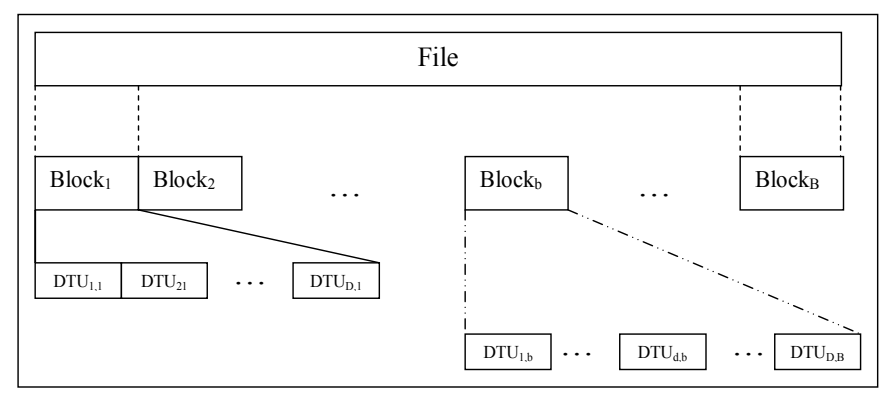

Figure 2. Relationship between file, block and DTU

\section{Channel-Aware Scheduling Algorithms WITH CHANNEL PREDICTION}

\section{A. Channel-Aware Scheduling (CAS) Algorithm}

According to CAS algorithm as defined and optimized in [4], a transmission of a packet in the forward link is resumed when the terminals are in good channel condition. Otherwise, the transmission is delayed by one slot. This operation can be described by inequality (1). In other words, if inequality (1) is fulfilled, then the packet is transmitted in the current forward bearer slot. Otherwise, the packet is delayed.

prtx $\leq$ prtxthresh

where the probability of retransmission, prtx, is calculated as

$p r t x=1-\prod_{j=1}^{N}\left(1-p_{j}\right)$

where $p_{j}$ is CSI value defined as the slot error rate from terminal $j$ and $N$ is the total number of terminals. The threshold, prtxthresh, is defined as

prtxthresh $=1-\left(1-\bar{p}_{j}\right)^{N}$

where $\bar{p}_{j}$ is average slot error rate. As shown in equations (2) and (3), both scheduling parameters prtx and prtxthresh are 
calculated from updated CSI value, $\bar{p}_{j}$, by the terminals. The parameter prtx represents instantaneous probability of retransmission of the packet, whereas prtxthresh corresponds to the long-term average of the probability of retransmission. In general, a lower prtx compared to prtxthresh signifies a better channel condition, resulting in higher probability of the packet to be received correctly by the terminals.

\section{B. CSI Collection and Suppression Policy}

At the terminal, CSI collection and suppression policy according to [5] is run. The operation starts with CSI measurement and comparison, which is executed every $10 \mathrm{~ms}$. The comparison employs change detection concept; if a significant change in CSI is observed, then the terminal will try to access the uplink slots for CSI updates. Otherwise, the terminals do not observe large variation of channel conditions worth accessing the slots for. In this case, the terminal is suppressed. This mechanism is implemented to avoid feedback implosion problem which is well known in multicast transmission.

This policy involves two slot error rate values from the terminals; the first is slot error rate averaged over a sliding observation window of length $V$ seconds, and the other one from an increasing reference window of length $t-V$ seconds. The two slot error rate values are compared at every CSI measurement; i.e., every $10 \mathrm{~ms}$. Upon detecting discrepancy between the two by a threshold value $\psi$, then the terminal is ready to transmit its CSI values. If not, the terminal is suppressed from accessing the uplink slots. In the simulations, the size of an observation window, $V$, is $80 \mathrm{~ms}$, and change detection threshold, $\psi$, is 0.05 .

\section{Uplink Access Scheme}

Upon CSI measurement and collection according to Section $\mathrm{B}$, a terminal that satisfies the policy is now ready to transmit its CSI values. The terminal randomly access contention slots (i.e., slots reserved for random access) to send a capacity request by sending a STATUS SDU (Signalling Data Unit) to the RNC to request scheduled slots. The STATUS SDU expresses the amount of data waiting in the terminal output buffers to be transmitted. If more than one terminal selects the same contention slot, a collision takes place on the uplink. Because of the collision, the transmitted STATUS SDU in this slot will be lost.

In order to reduce the probability of collision, terminals randomly select a contention slot. If the STATUS SDU is correctly received by the RAN, then, in response, the RAN allocates a reservation slot for the terminal. The terminal then utilizes the allocated reservation slot for its CSI update. Apart from CSI updates, the reservation slots is also used for higherlayer traffic, such as NACKs and DONE messages. The reception of STATUS SDUs must be confirmed by the STATUS-ACK SDU from the RNC. If the terminal does not receive a STATUS-ACK before the STATUS-ACK-TIMER expires, the terminal repeats the random contention slot selection procedure to send a new STATUS SDU.

The uplink capacity for CSI updates from the terminals is organized in 80ms MF-TDMA frames that are composed of a number of $5 \mathrm{~ms}$ and $20 \mathrm{~ms}$ time slots. In the simulation, three uplink carriers are used, 2 carriers consists of four $20 \mathrm{~ms}$ reservation slot each, and one carrier consists of $165 \mathrm{~ms}$ contention slots.

\section{Real-time Channel Prediction Technique (RTChP)}

At the scheduler in RAN, successful CSI updates arrive according to CSI collection and suppression policy described in Section II-B. Based on the updated CSI, real-time channel prediction (RTChP) is considered to improve the performance of CAS by tracking the behavior of scheduling parameter prtx in time. The operation of RTChP (or lack of it) is described as follows:

i) First, the scheduler calculates the scheduling parameter prtx according to equation (2).

ii) Next, with RTChP in place, prtx is predicted $\frac{t_{R T T}}{2}$ ahead. Based on the predicted value, the scheduler decides to use or not the current slot according to inequality (1). In contrast, without RTChP in place, prtx is calculated merely based on $p_{j}(t)$ updated from the terminals.

The channel estimation technique that is used in this context is derived from [6], which is claimed to be more accurate and reliable estimation technique pertaining to satellite channels. In our simulation, the scheduling parameters prtx is sampled every $n_{0}=\frac{t_{R T T}}{2} \mathrm{~s}$. At sample index $n=n_{t r}$, we want to predict the value of prtx in the future, i.e., at arrival correspond to $n=n_{t r}+n_{0}$. Thus, the general prediction is

$\hat{p r t x}\left[n_{t r}+n_{0}\right]=c_{1} \operatorname{prtx}\left[n_{t r}\right]+c_{2} \operatorname{prtx}\left[n_{t r}-1\right]$

where $n_{0} \geq 0$ represents how far ahead we want the predictor to tell the behavior of prtx in the future, in our case $n_{0}=\frac{t_{R T T}}{2}$; i.e. one sample away. AutoRegressive (AR) model with two poles are assumed, hence the number of past data used in prediction to that poles are restricted, i.e. one or two. The value of prediction coefficients $c_{1}$ and $c_{2}$ can be resolved in a matrix form. In the case of 2-pole Yule-Walker, prediction coefficient $c$ can be presented in matrix form as

$$
\left[\begin{array}{cc}
K_{p p}[0] & K_{p p}[1] \\
K_{p p}[1] & K_{p p}[0]
\end{array}\right]\left[\begin{array}{l}
c_{1} \\
c_{2}
\end{array}\right]=\left[\begin{array}{c}
K_{p p}\left[1+n_{0}\right] \\
K_{p p}\left[2+n_{0}\right]
\end{array}\right]
$$


where $K_{p p}[\mathrm{~m}]$ is correlation function of past data. To solve the inverse of matrix (7), $K_{p p}[m]$ must be estimated itself. In reference [6] it is suggested that estimate of $K_{p p}[m], \hat{K}_{p p}[m]$, can be calculated using sample correlation function from past data. Basically, the predictor uses the last $X$ data points to obtain estimated value of $p r t x, \hat{K}_{p p}$ :

$\hat{K}_{p p}[m, q]= \begin{cases}\frac{1}{X-|m|} \sum_{a=q-X+1}^{q-|m|} \operatorname{prtx}[a] \operatorname{prtx}[a+|m|] & \text { if } a \leq X-\mid m \\ 0 & \text { otherwise }\end{cases}$

In our consideration, the last $X=10$ last data points of prtx is used. From the estimation of of $K_{p p}[\mathrm{~m}]$ in equation (8), the prediction coefficients $c_{1}$ and $c_{2}$ at each $n_{t r}$ can be obtained, which are then used to predict $\hat{p} r t x\left[n_{t r}+n_{0}\right]$ according to equation (6).

Likewise, a scenario is compared where RTChP is not implemented. In such scenario, the scheduling parameter prtx considers only the CSI values updated from the terminals, i.e. without using prediction technique. Subsequently, the value of prtx becomes:

$\hat{p} r t x\left[n_{t r}+n_{0}\right]=\operatorname{prtx}\left[n_{t r}\right]$

TABLE I SIMULATION PARAMETERS

\begin{tabular}{|l|l|}
\hline \multicolumn{1}{|c|}{ Parameter } & \multicolumn{1}{c|}{ Value } \\
\hline Number of terminals, $N$ & $5120,15,20\}$ \\
\hline Payload size, $L$ & $10 \mathrm{~ms}$ \\
\hline Payload duration, $t_{\text {Slot }}$ & $10 \mathrm{MB}$ \\
\hline File size, $F$ & $800 \mathrm{~ms}$ \\
\hline Round-trip-time, $t_{R T T}$ & 11392 bits \\
\hline DTU payload size, $L_{D T U}$ & 7364 DTUs \\
\hline $\begin{array}{l}\text { Total number of DTUs in the } \\
\text { file, } M\end{array}$ & $\begin{array}{l}8 \text { reservation slots, } 16 \\
\text { contention slots }\end{array}$ \\
\hline Uplink slots & $80 \mathrm{~ms}$ \\
\hline Change detection parameter, $V$ & 0.05 \\
\hline Change detection threshold, $\psi$ & $\begin{array}{l}6.98 \text { dB } \\
(\text { Std. deviation }=1.69 \mathrm{~dB})\end{array}$ \\
\hline Average $E_{b} / N_{o}$ & $2.25 e-4$ \\
\hline Average slot error rate, $\bar{p}$ & $\begin{array}{l}\text { Mean }=0.98 \mathrm{~s} \\
(\text { Std. deviation }=1.45 \mathrm{~s})\end{array}$ \\
\hline Log-normal fading duration & $\begin{array}{l}\text { Mean }=1.37 \mathrm{~s} \\
(\text { Std. deviation }=2.22 \mathrm{~s})\end{array}$ \\
\hline Power law LOS duration & \\
\hline
\end{tabular}

\section{PERFORMANCE ANALYSIS}

To observe the scheduling performance with respect to implementation of RTChP, simulations of reliable transmission of a file with size $F$ MB to $N$ number of terminals are run. In the simulation analysis, the parameters listed in Table I are observed.

The performance of RTChP is tested against number of terminals. In Figures 3 and 4, the average session duration and number of DTUs retransmitted are plotted against number of terminals, $N$, respectively. As shown in the figures, it is found that both session duration and number of DTUs retransmitted observe better performance with RTChP in place when few terminals are considered. As number of terminals is increased, RTChP becomes ineffective in predicting the future value of scheduling parameter $\hat{p} r t x$, resulting in worse performance. This is because RTChP is implemented at the scheduler in RAN, rather than at each terminal. It is expected that if RTChP is run at the terminal to predict its CSI value (i.e. $p$ ) one roundtrip time away, then the performance will be greatly improved. The only constraint for such implementation is that complexity increases, since we are considering multicast scenario. Furthermore, the limited number of contention and reservation slots resulting in higher number of collisions when more terminals are trying to access the uplink slots for CSI updates. This is illustrated in Figure 5, where the number of collision increases when number of terminals increases.

Certainly, the RTChP scheme is not perfect, but knowledge of future channel condition might greatly help with the scheduling decision which results in higher accuracy and reliability of the decision, particularly when small number of terminals is considered. As more terminals are expected especially in geostationary satellite networks, the RTChP might not be effective in coping with terminal heterogeneity.

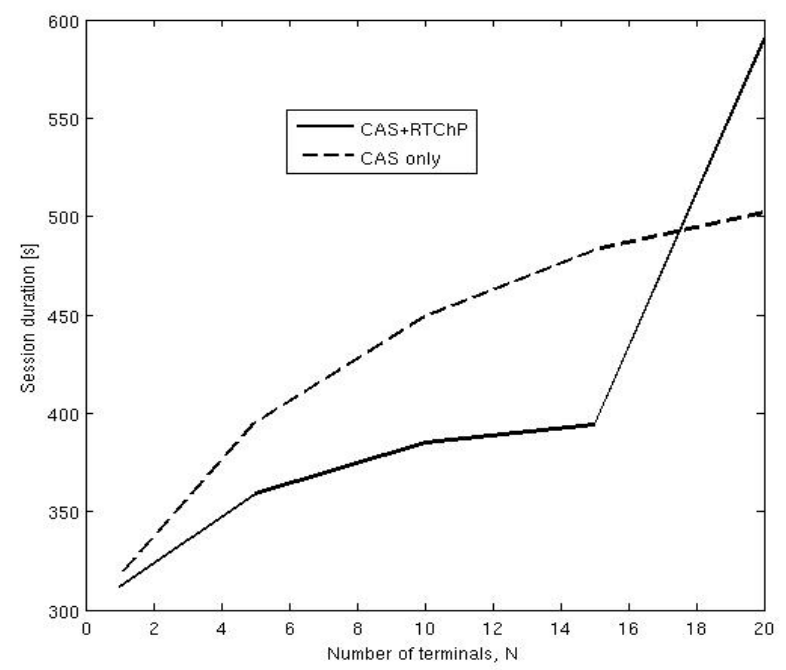

Figure 3. CAS algorithm, session duration vs. $N$, with and without real-time channel prediction technique (RTChP) 


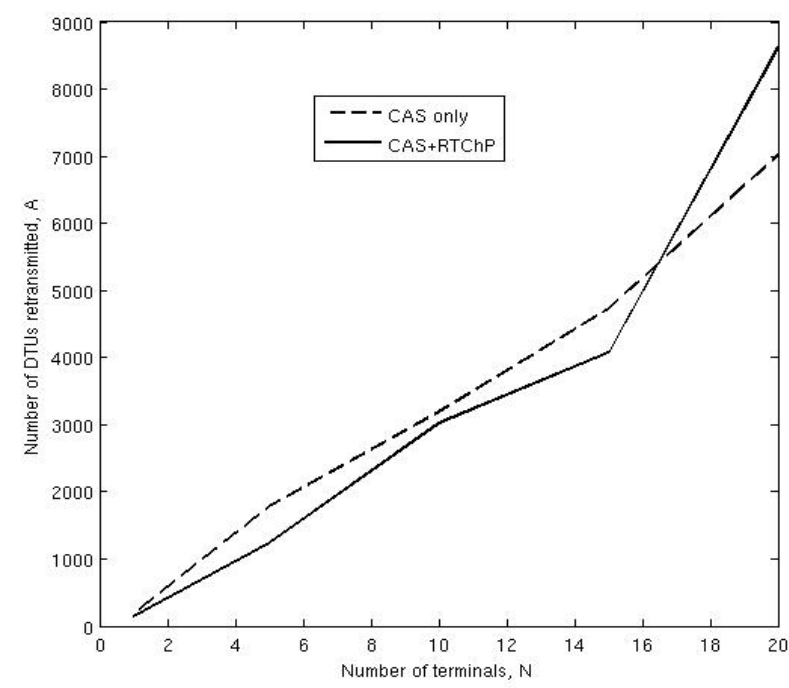

Figure 4. CAS algorithm, number of DTUs retransmitted vs. $N$, with and without real-time channel prediction technique (RTChP)

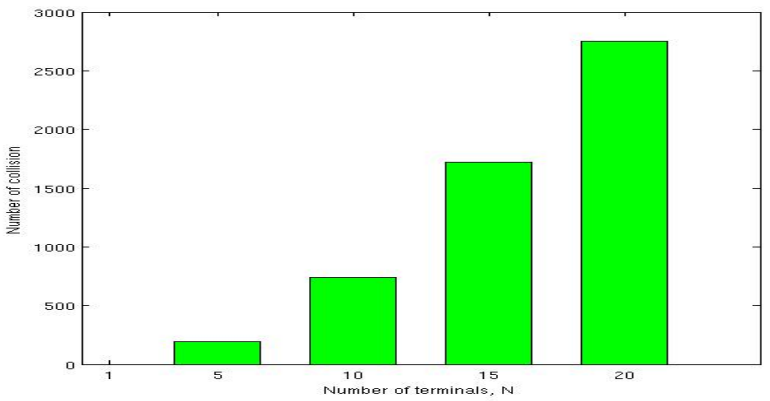

Figure 5. Number of collision vs. number of terminals, $N$

\section{CONCLUSION}

In this paper we show the possible implementation of channel-aware scheduling algorithms with channel prediction technique in the spirit of cross-layer design to improve the performance of reliable multicast transmissions over geostationary satellite channels. In particular, interactions between CSI values from PHY layer and channel-aware scheduler in MAC layer are investigated in the presence of $L$ band satellite channel.

A scheduling algorithm is studied, called Channel-Aware Scheduler (CAS). Based on CAS algorithm, RTChP is run upon, with the objective to increase the accuracy of scheduling decision. It is found that session delay and number of packets retransmitted are greatly reduced with RTChP in place for low and medium number of terminals.

Currently, channel-aware scheduling algorithm is tested on single multicast data product. It will be interesting to investigate the scheduling mechanism when there is more than one multicast group is considered, each terminal in the group experiencing different channel condition. Such scenario might call for optimized solution, which is our future study of interest.

\section{ACKNOWLEDGMENT}

This paper has been carried out within the framework of European SatNEx II (contract no. IST-027393) network of excellence, www.satnex.org, joint activity JA2330 "Resource Allocation and Adaptation".

\section{REFERENCES}

[1] G. Giambene, S. L. Kota, "Cross-Layer Protocol Optimization for Satellite Communications Networks: A Survey", International Journal of Satellite Communications and Networking, Vol. 24, pp. 323-341, 2006.

[2] L. E. Braten, T. Tjelta, "Semi-Markov Multistate Modeling of Land Mobile Propagation Channel for Geostationary Satellites", IEEE Transactions on Antennas and Propagation, Vol. 50, No. 12, December 2002.

[3] K. Miller, K. Robertson, "StarBurst Multicast File Transfer protocol (MFTP) Specification", IETF Draft <draft-miller-mftp-spec-03.txt>, July 1998.

[4] A. Sali , G. Acar, B. Evans, "A Cross-Layer Approach for Scheduling in reliable Multicast Data Transmission over Geostationary Satellite Networks", IEEE 65th Vehicular Technology Conference VTC2007, Dublin, $23^{\text {rd }}-25^{\text {th }}$ April 2007.

[5] A. Sali, G. Acar, B. Evans, G. Giambene, 'Change Detection Mechanism in Feedback Implosion Suppression Algorithm for Reliable Transmission of Multicast Data over Geostationary Satellite Network', 26th AIAA International Communications Satellite Systems Conference (ICSSC-2008), San Diego, $10^{\text {th }}-12^{\text {th }}$ June 2008

[6] J. P. Choi, V. W. S. Chan, "Predicting and Adapting Satellite Channels with Weather-Induced Impairments", IEEE Transactions on Aerospace and Electronics Systems, Vol. 38, No. 3, July 2002. 